\title{
Hadronic Charm Decays at BESIII
}

\author{
Shuaiying $\mathbf{L i}^{* \dagger}$ \\ for the BESIII Collaboration \\ Department of Engineering Physics, Tsinghua University, Beijing, China \\ E-mail: lishy@ihep.ac.cn
}

The BESIII experiment at the Beijing Electron Positron Collider (BEPCII) has accumulated the world's largest samples of $e^{+} e^{-}$collisions in the tau-charm region. Based on the threshold open charm data samples of $D_{(s)}$ and $\Lambda_{c}^{+}$, we can study the hadronic charm decays under a uniquely clean background. Many important results have been achieved with much-improved precision or for the first time. In this paper, we will review some recent results on the hadronic charm decays. Finally, a summary will be given.

XXIX International Symposium on Lepton Photon Interactions at High Energies - LeptonPhoton2019 August 5-10, 2019

Toronto, Canada

\section{* Speaker.}

${ }^{\dagger}$ I would like to thank for the support by the National Natural Science Foundation of China (Grants No. 11575094). 


\section{Introduction}

Except for a single tag (ST) technique used in Sec. 2.6, all other items use a double tag (DT) technique to perform event selection. DT means reconstructing both $D_{s}^{+}\left(\Lambda_{c}^{+}\right)$and $D_{s}^{-}\left(\Lambda_{c}^{-}\right)$, while ST means reconstructing only one $D_{s}^{+}\left(\Lambda_{c}^{+}\right)$or $D_{s}^{-}\left(\Lambda_{c}^{-}\right)$. DT technique provides a clean environment with no additional hadron and also provides access to absolute branching fractions (BFs). ST technique can achieve more data yields.

Besides, the variable Missing-mass-squared $\left(M M^{2}\right)$ mentioned below are defined here.

$M M^{2}=\left(P_{e^{+} e^{-}}-P_{D_{s}^{-}}-P_{\gamma}-P_{K^{+}}\right)^{2}$, where $P_{e^{+} e^{-}}$is the four-momentum of the $e^{+} e^{-}$initial state and $P_{i}\left(i=D_{s}^{-}, \gamma, K^{+}\right)$is the four-momentum of the corresponding particle.

\section{Hadronic Charm Decays}

There are seven analyses listed below. The first four items are about $D_{s}$ decays, which is based on $3.19 \mathrm{fb}^{-1}$ data set accumulated at $E_{c m}=4.18 \mathrm{GeV}$ by BESIII detector in 2016 . And the rest three items are about $\Lambda_{c}$ decays, which is based on $567 p b^{-1}$ data sample taken at $E_{c m}=4.6 \mathrm{GeV}$ with the BESIII detector from February to March of 2014.

2.1 $D_{s}^{+} \rightarrow \omega \pi^{+} / K^{+}$

This is a pure $\mathrm{W}$ annihilation process, which is sensitive to direct $\mathrm{CP}$ violation. We obtain $65.0 \pm 11.6 D_{s}^{+} \rightarrow \omega \pi^{+}$signal events and $28.5 \pm 7.8 D_{s}^{+} \rightarrow \omega K^{+}$signal events with statistical significances of $6.7 \sigma$ and $4.4 \sigma$, respectively. The BF of $D_{s}^{+} \rightarrow \omega \pi^{+}$is $\mathscr{B}_{D_{s}^{+} \rightarrow \omega \pi^{+}}=$ $\left(1.77 \pm 0.32_{\text {stat. }} \pm 0.13_{\text {sys. }}\right) \times 10^{-3}$ which is consistent with CLEO's measurement [1], but more precise. The $\mathrm{BF}$ of $D_{s}^{+} \rightarrow \omega K^{+}$is $\mathscr{B}_{D_{s}^{+} \rightarrow \omega K^{+}}=\left(1.77 \pm 0.32_{\text {stat. }} \pm 0.13_{\text {sys. }}\right) \times 10^{-3}$ which is the first evidence [2].

$2.2 D_{s}^{+} \rightarrow p \bar{n}$

This is the only kinematically allowed hadronic decay involving baryons. The short-distance contribution is expected to be small: $\mathrm{BF} \sim 10^{-6}$, due to the chiral suppression by a factor of $\left(m_{\pi} / m_{D_{s}}\right)^{4}$. But long-distance effects can enhance the BF to a level of $10^{-3}$ [3]. We obtain $193 \pm 17$ $D_{s}^{+} \rightarrow p \bar{n}$ signal events. And the absolute $\mathrm{BF}$ is $\mathscr{B}_{D_{s}^{+} \rightarrow p \bar{n}}=\left(1.21 \pm 0.10_{\text {stat. }} \pm 0.05_{\text {sys. }}\right) \times 10^{-3}$ [4]. The first evidence was reported by CLEO with a signal of $13.0 \pm 3.6$ events with $\mathrm{BF} \mathscr{B}_{D_{s}^{+} \rightarrow p \bar{n}}=$ $\left(1.30 \pm 0.36 \pm_{0.16}^{0.12}\right) \times 10^{-3}[5]$.

\section{3 $D_{S}^{+} \rightarrow K_{S}^{0} K^{+}$and $K_{L}^{0} K^{+}$}

The interference of the decay amplitudes of the Cabibbo-favored (CF) transition $D \rightarrow \bar{K}^{0} K$ and the doubly-Cabibbo-suppressed (DCS) transition $D \rightarrow K^{0} K$ can result in a measurable $K_{S}^{0}-$ $K_{L}^{0}$ asymmetry. Additionally, as pointed out in Ref. [6], the interference between CF and DCS amplitudes can also lead to a new CP violation effect, which is estimated to be an order of $10^{-3}$.

The $2 \mathrm{D}$ fit for $D_{s}^{+} \rightarrow K_{S}^{0} K^{+}$gives a signal yield of $1782 \pm 47$. And the projections of $M_{K_{S}^{0} K^{+}}$ with the fit results superimposed are shown in Fig. 1(a,b). The fit to the $M M^{2}$ distribution is shown in Fig. 1(c). And the signal yield determined by the fit is $2349 \pm 61$ events. The absolute BFs results 
are $\mathscr{B}_{D_{s}^{+} \rightarrow K_{S}^{0} K^{+}}=(1.425 \pm 0.038 \pm 0.031) \%$ and $\mathscr{B}_{D_{s}^{+} \rightarrow K_{L}^{0} K^{+}}=(1.485 \pm 0.039 \pm 0.046) \%$. The branching fraction of $D_{s}^{+} \rightarrow K_{S}^{0} K^{+}$is compatible with the world average and that of $D_{s}^{+} \rightarrow K_{L}^{0} K^{+}$ is measured for the first time.

We also measure the direct CP asymmetries of $D_{s}^{ \pm} \rightarrow K_{S}^{0} K^{ \pm}$and $D_{s}^{ \pm} \rightarrow K_{L}^{0} K^{ \pm}$. Moreover, we present the first measurement of the $K_{S}^{0}-K_{L}^{0}$ asymmetry in the decays $D_{s}^{+} \rightarrow K_{S, L}^{0} K^{+}$. The results are all consistent with zero [7].

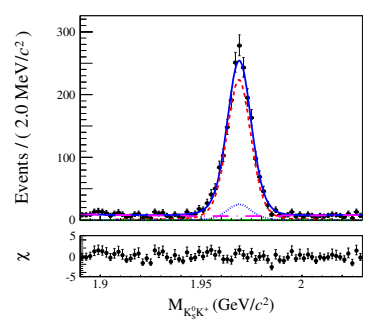

(a)

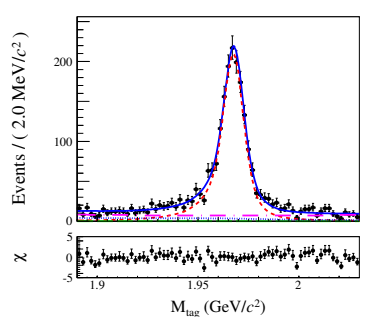

(b)

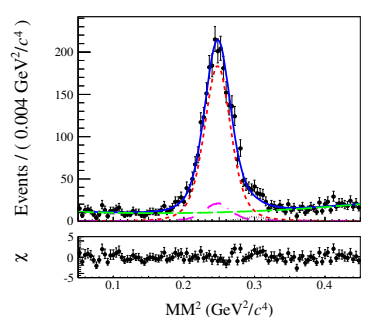

(c)

Figure 1: Distributions of $M_{K_{S}^{0} K^{+}}$(a), $M_{t a g}$ (b), and $M M^{2}$ (c), with the fit result superimposed. The data are shown as the dots with error bars, the blue solid line is the total fit result, the red short-dashed line is the signal component of the fit. The green long-dashed line, the blue dotted line and the magenta dotted-dashed line in (a) and (b) are the three background components mentioned in [7]. The magenta dotted-dashed line, the grey dotted line and the green long-dashed line in (c) are the component of the peaking background from $D_{s}^{+} \rightarrow K_{S}^{0} K^{+}$decays, $D_{s}^{+} \rightarrow \eta K^{+}$decays, and non-peaking background component, respectively. The residual $\chi$ between the data and the total fit result, normalized by the uncertainty, is shown beneath the figures.

\subsection{Ampitude analysis of $D_{s}^{+} \rightarrow \pi^{+} \pi^{0} \eta$}

We retain a sample of $1239 D_{s}^{+} \rightarrow \pi^{+} \pi^{0} \eta$ candidates that has a purity of $(97.7 \pm 0.5) \%$. We observe for the first time the pure $\mathrm{W}$-annihilation decays $D_{s}^{+} \rightarrow a_{0}(980)^{+} \pi^{0}$ and $D_{s}^{+} \rightarrow a_{0}(980)^{0} \pi^{+}$. We measure the absolute BFs $\mathscr{B}_{D_{s}^{+} \rightarrow a_{0}(980)^{+(0)} \pi^{0(+)}, a_{0}(980)^{+(0)} \rightarrow \pi^{+(0)} \eta}=(1.46 \pm 0.15 \pm 0.23) \%$, which is larger than the BFs of other measured pure $\mathrm{W}$-annihilation decays by at least one order of magnitude [8]. The fit projections are shown in Fig. 2.
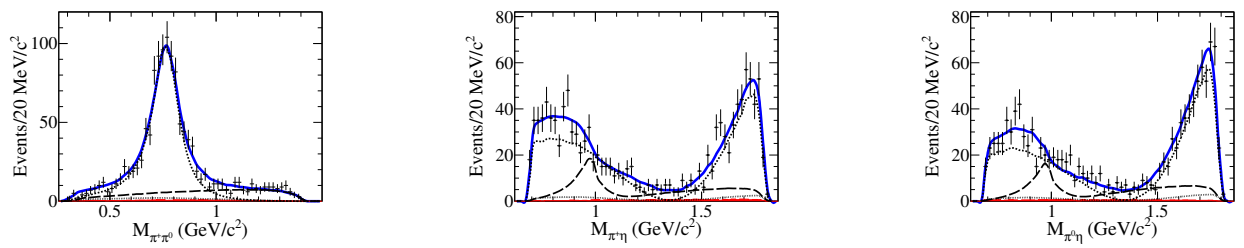

Figure 2: The dots with error bars and the solid line are data and the total fit, respectively. The dashed, dotted, and long-dashed lines are the contributions from $D_{s}^{+} \rightarrow \rho^{+} \eta, D_{s}^{+} \rightarrow\left(\pi^{+} \pi^{0}\right)_{V} \eta$, and $D_{s}^{+} \rightarrow a_{0}(980) \pi$, respectively.

\section{$2.5 \Lambda_{c}^{+} \rightarrow \Lambda X$}

We measure the absolute BF of the inclusive decay of $\Lambda_{c}^{+} \rightarrow \Lambda X$ to be $\mathscr{B}_{\Lambda_{c}^{+} \rightarrow \Lambda X}=\left(38.2 \pm_{2.2}^{2.8}\right.$ $\pm 0.6) \%$ using the double tag method. Also, we search for direct CP violation in the charge asymmetry of this inclusive decay for the first time. The result is consistent with zero [9]. 
$2.6 \Lambda_{c}^{+} \rightarrow \Lambda \eta \pi^{+}$and $\Lambda_{c}^{+} \rightarrow \Sigma(1385)^{+} \eta$

We obtain $154 \pm 17 \Lambda_{c}^{+} \rightarrow \Lambda \eta \pi^{+}$events and $54 \pm 11 \Lambda_{c}^{+} \rightarrow \Sigma(1385)^{+} \eta$ events with a ST technique. And the BFs are measured to be $\mathscr{B}_{\Lambda_{c}^{+} \rightarrow \Lambda \eta \pi^{+}}=(1.84 \pm 0.21 \pm 0.15) \%$ and $\mathscr{B}_{\Lambda_{c}^{+} \rightarrow \Sigma(1385)^{+} \eta}=$ $(0.91 \pm 0.18 \pm 0.09) \%$, constituting the most precise measurements to date [10].

$2.7 \Lambda_{c}^{+} \rightarrow \Sigma^{+} \eta / \eta^{\prime}$

They are CF decays, which proceed through non-factorizable internal W-mission. We find evidence for the decays $\Lambda_{c}^{+} \rightarrow \Sigma^{+} \eta$ and $\Lambda_{c}^{+} \rightarrow \Sigma^{+} \eta^{\prime}$ with statistical significance of $2.5 \sigma$ and $3.2 \sigma$, respectively. Using BESIII measurements of the BFs of the reference decays, we determine $\mathscr{B}_{\Lambda_{c}^{+} \rightarrow \Sigma^{+} \eta}=(0.41 \pm 0.19 \pm 0.05) \%(<0.68 \%$ at $90 \%$ C.L. $)$ and $\mathscr{B}_{\Lambda_{c}^{+} \rightarrow \Sigma^{+} \eta^{\prime}}=(1.34 \pm 0.53 \pm$ $0.19) \%(<1.9 \%$ at $90 \%$ C.L. $)$. The BF of $\Lambda_{c}^{+} \rightarrow \Sigma^{+} \eta$ is consistent with the previous measurement, and the BF of $\Lambda_{c}^{+} \rightarrow \Sigma^{+} \eta^{\prime}$ is measured for the first time [11].

\section{Summary}

Our results include new measurements, have confirmed and improved the precisions over the previous results. We are planning to take more data at/near $E_{c m}=4.6 \mathrm{GeV}$ as well as $E_{c m}=$ $3.773 \mathrm{GeV}$ soon, which will allow us to even improve further precisions and rare forbidden searches in $D_{(s)} / \Lambda_{c}$ decays. More measurements in $D_{(s)} / \Lambda_{c}$ hadronic decays are coming.

\section{References}

[1] J. Y. Ge et al. (CLEO Collaboration), Phys. Rev. D 80, 051102(R) (2009).

[2] M. Ablikim et al. (BESIII Collaboration),Phys. Rev. D 99, 091101(R) (2019).

[3] Chuan-Hung Chen, Hai-Yang Cheng, and Yu-Kuo Hsiao, Phys. Lett. B 663, 326 (2008).

[4] M. Ablikim et al. (BESIII Collaboration), Phys. Rev. D 99, 031101(R) (2019).

[5] S. B. Athar et al. (CLEO Collaboration), Phys. Rev. Lett. 100, 181802 (2008).

[6] Di Wang, Fu-Sheng Yu, and Hsiang-nan Li, Phys. Rev. Lett. 119, 181802 (2017).

[7] M. Ablikim et al. (BESIII Collaboration), Phys. Rev. D 99, 112005 (2019).

[8] M. Ablikim et al. (BESIII Collaboration), arXiv:1903.04118 (2019).

[9] M. Ablikim et al. (BESIII Collaboration), Phys. Rev. Lett. 121, 062003 (2018).

[10] M. Ablikim et al. (BESIII Collaboration), Phys. Rev. D 99, 032010 (2019).

[11] M. Ablikim et al. (BESIII Collaboration), Chin. Phys. C 43, 083002 (2019). 